\title{
Dor de dente como preditor de absenteísmo em trabalhadores de uma indústria de sucos da Região Sudeste do Brasil
}

\author{
Dental pain as a predictor of absenteeism among workers \\ in a juice factory in southeastern Brazil
}

Maria Helena Monteiro de Barros Miotto ${ }^{1}$

Ludmilla Awad Barcellos ${ }^{2}$

Zulmara Vicentini Lopes ${ }^{3}$

\footnotetext{
${ }^{1}$ Departamento de Clínica Odontológica, Centro Biomédico, Universidade Federal do Espírito Santo. Av. Marechal Campos 1468, Maruípe. 29.040-090

Vitoria ES Brasil. mhmiotto@terra.com.br

${ }^{2}$ Universidade de Vila Velha.

${ }^{3}$ Associação Brasileira de Odontologia.
}

\begin{abstract}
Pain is a public health problem responsible for loss of work days. The scope of this article is to analyze the prevalence of dental pain and absenteeism, and the possible association with social and demographic characteristics. This crosssectional study involved a random sample of 169 individuals selected from a universe of 666 workers. Data was collected by a trained researcher using a structured questionnaire. Fisher's Exact Test verified the possible associations; the strength of the associations was verified by the Odds Ratio with 5\% significance. The prevalence of dental pain was $46.7 \%$, and absenteeism $12.7 \%$. There was no association between dental pain and the variables analyzed, namely sex, age, income and education. With respect to absenteeism, the individuals with less years of instruction declared greater loss of work hours $(O R=8.850$, IC $95 \%=2.114$; 37.046). The prevalence of pain was considerable and was not associated with the controlled variables of this study. Dental pain observed was sufficient to lead to absenteeism, and this was associated with education. Periodic exams must be encouraged for diagnosis and early intervention, thereby minimizing episodes of dental pain.

Key words Dental pain, Absenteeism, Occupational health, Public health
\end{abstract}

Resumo Dor é um problema de saúde pública, responsável pela perda de dias de trabalho. O objetivo deste artigo é analisar a prevalência da dor dentária, a ocorrência de absenteísmo e suas possíveis associações com características sociodemográficas. Este estudo transversal utilizou uma amostra aleatória de 169 sujeitos de um universo de 666 funcionários. Dados foram coletados por pesquisadora treinada utilizando roteiro estruturado. O teste exato de Fisher avaliou as possíveis associações; a força da associação avaliada pelo Odds Ratio com significância de 5\%. A prevalência de dor dentária foi de 46,7\% e a de absenteísmo 12,7\%. Não houve associação estatística entre dor e as variáveis avaliadas - sexo, faixa etária, renda e escolaridade. Em relação ao absenteísmo, indivíduos com grau de escolaridade até o ensino médio incompleto declararam maior perda de horas de trabalho $(O R=8,850, I C 95 \%=$ $2,114 ; 37,046)$. A prevalência de dor foi expressiva e não esteve associada às variáveis controladas neste estudo. A dor de dente observada foi capaz de produzir absenteísmo, e este esteve associado à escolaridade dos participantes. A realização de exames periódicos odontológicos deve ser incentivada para diagnóstico e intervenção precoce, minimizando episódios de dor de dente.

Palavras-chave Dor dentária, Absenteísmo, Saúde do trabalhador, Saúde pública 


\section{Introdução}

Dor é um problema de saúde pública, que provoca um impacto significante na vida dos indivíduos e na sociedade como um todo, podendo ser responsável pela perda de muitos dias de traba1 ho ${ }^{1}$. Milhões de pessoas em todos os países ainda sofrem de dor de dente ${ }^{2}$. Estudos nacionais têm revelado uma alta prevalência de dor de dente entre os adultos ${ }^{3}$. Diversos estudos realizados no Espírito Santo revelaram alta prevalência de absenteísmo associado à dor de dente entre trabalhadores de vários setores da economia ${ }^{4-6}$.

Absenteísmo é um tema de crescente interesse por sua importância econômica e competitividade entre as empresas, que buscam diminuir sua ocorrência, possibilitando, assim, uma maior produtividade ${ }^{7}$. Portanto, é importante para a economia de um país a preservação e a conservação da saúde do trabalhador por interferir diretamente na capacidade produtiva ${ }^{8}$. No Brasil, não existem valores aferidos de dias perdidos do trabalho por razões de absenteísmo odontológico, em nível nacional, estadual ou municipal. Não há informações a respeito do ônus econômico que as faltas possam acarretar como também sobre o nível de insatisfação do trabalhador pela quebra do binômio saúde/trabalho9.

Milhares de sujeitos podem ter suas atividades laborais diárias, inclusive de lazer, prejudicadas, em decorrência de problemas de dor de origem dentária tanto provocada como espontânea ${ }^{10,11}$. Em Recife, uma das dimensões mais afetadas entre adolescentes e suas famílias foi a execução das atividades diárias domésticas ${ }^{12}$. Portanto, o reconhecimento dos problemas bucais que podem impactar a qualidade de vida dos trabalhadores é importante no planejamento de serviços odontológicos ${ }^{13}$.

O objetivo deste estudo foi analisar a prevalência da dor dentária nos últimos seis meses e a ocorrência de absenteísmo provocado por dor de dente, além de possíveis associações com as características sociodemográficas, em uma amostra de trabalhadores de uma indústria alimentícia em um município do Norte do Estado do Espírito Santo.

\section{Metodologia}

Este estudo populacional, observacional, analítico e transversal utilizou uma amostra representativa dos funcionários da maior indústria alimentícia do município de Linhares Estado do Espírito Santo, Brasil.
Uma listagem cedida pelo Setor Recursos Humanos da empresa revelou um quadro de 666 funcionários divididos em três turnos de trabalho. O cálculo amostral resultou em 170 trabalhadores, tendo como parâmetros uma prevalência esperada de $30 \%$, nível de confiança de $95 \%$ e erro igual a $5 \%$. Foi extraída uma amostra aleatória de 170 sujeitos acrescida de 40 funcionários, prevendo a possibilidade de perda amostral.

O critério de inclusão adotado foi constar o nome na lista de funcionários em plena atividade. Não foram incluídos trabalhadores afastados por licença médica e terceirizados.

Foi realizado um projeto piloto com uma amostra de 15 funcionários da própria indústria, excluídos do estudo principal, para possibilitar ajustes.

Um roteiro estruturado com 29 questões fechadas foi utilizado para a coleta de dados em 2007, realizada por profissionais treinadas, sendo uma auxiliar em saúde bucal, e duas técnicas de enfermagem do Serviço Especializado em Engenharia de Segurança e Medicina do Trabalho (SESMT) da empresa. O questionário foi autogerenciado e aplicado nos três turnos de trabalho, após a obtenção do Termo de Consentimento Livre e Esclarecido.

Para o estudo foram considerados dentados indivíduos com pelo menos um dente presente; necessidade autorreferida de prótese parcial removível foi declarada por indivíduos com perda de alguns elementos dentários; necessidade autorreferida de prótese total removível para aqueles que declararam ausência total de dentes em uma das arcadas dentárias.

A comparação dos percentuais entre dor dentária e absenteísmo por dor dentária com os fatores sociodemográficos foi efetivada pelo teste exato de Fisher com significância de 5\%. Foram calculados o Odds Ratio (OR) e os respectivos intervalos de confiança.

A pesquisa foi conduzida dentro dos padrões exigidos pela Resolução no 196/96 $6^{14}$, aprovada, em julho de 2007, pelo Comitê de Ética em Pesquisa da Associação Brasileira de Odontologia, ES.

\section{Resultados}

A amostra final deste estudo contou com 169 sujeitos, uma perda considerada inexpressiva em virtude do cálculo amostral de 170 indivíduos. O acréscimo de $20 \%$ foi suficiente para garantir o poder amostral. A recusa não comprometeu a representatividade em nenhum subgrupo. Os 
participantes eram principalmente do sexo masculino $(66,3 \%)$, jovens até 30 anos $(74 \%)$, com escolaridade acima do ensino médio completo $(83,4 \%)$ e $46,2 \%$ com renda declarada de até dois salários mínimos (Tabela 1).

A grande parte dos entrevistados (47,4\%) relatou possuir todos os dentes; não houve edentados na amostra. Em relação à necessidade percebida de prótese, apenas 7,7\% declararam precisar de Prótese Parcial Removível (PPR) e 1,2\% de Prótese Total Removível (PTR) em uma das arcadas (Tabela 2).

Foi verificada uma alta prevalência de dor dentária $(46,7 \%)$. Desses, 45 (57\%) visitaram o cirurgião-dentista, originando $12,7 \%$ de falta ao trabalho, com $40 \%$ perdendo um turno de trabalho. Quase a metade dos trabalhadores $(48,1 \%)$ que sentiu dor de origem dentária compareceu ao trabalho. Dos trabalhadores que visitaram o dentista por dor de dente, a maior parte $(48,9 \%)$ utilizou o serviço odontológico da empresa e $40 \%$ procuraram o serviço privado ( $\mathrm{Ta}-$ bela 3 ).

A prevalência de dor de dente não esteve associada às variáveis sociodemográficas - sexo, faixa etária, escolaridade e renda (Tabela 4).

A prevalência de absenteísmo foi maior em indivíduos com menor grau de escolaridade $(\mathrm{p}=$ $0,004)$. Calculada a razão de chances, ficou demonstrado que os trabalhadores com menor escolaridade têm oito vezes maior chance de absenteísmo $(\mathrm{OR}=8,850$, IC95\% $=2,114 ; 37,046)$.

Tabela 1. Características sociodemográficas dos funcionários de uma empresa alimentícia em Linhares, ES, 2007.

\begin{tabular}{lrr}
\hline \multicolumn{1}{c}{ Característica } & n & $\%$ \\
\hline Sexo & 112 & 66,3 \\
$\quad$ Masculino & 57 & 33,7 \\
$\quad$ Feminino & & \\
Faixa Etária & 28 & 16,6 \\
Até 20 anos & 97 & 57,4 \\
21 - 30 anos & 40 & 23,7 \\
31 - 40 anos & 4 & 2,4 \\
41 anos ou mais & & \\
Nível de Escolaridade & 28 & 16,6 \\
$\quad$ Até ens. médio incompleto & 141 & 83,4 \\
$\quad$ Acima do ens. médio completo & & \\
Renda Familiar & 78 & 46,2 \\
$\quad$ Até 2 salários mínimos & 91 & 53,8 \\
$\quad$ Acima de 2 salários mínimos & 169 & 100,0 \\
Total &
\end{tabular}

Tabela 2. Situação da dentição declarada pelos funcionários de uma empresa alimentícia em Linhares, ES, 2007.

\begin{tabular}{lrr}
\hline \multicolumn{1}{c}{ Característica } & n & $\%$ \\
\hline Classificação & 169 & 100,0 \\
$\quad$ Dentado & 0 & 0 \\
$\quad$ Edentado & & \\
Perda Dentária & 80 & 47,4 \\
$\quad$ Nenhuma & 53 & 31,3 \\
Um dente a dois dentes & 36 & 21,3 \\
Três dentes ou mais dentes & & \\
Necessidade de prótese parcial removível & 147 & 87,0 \\
$\quad$ Não preciso usar & 13 & 7,7 \\
Preciso & 9 & 5,3 \\
$\quad$ Não sabe & & \\
Necessidade de prótese total removível & 164 & 97,0 \\
Não preciso usar & 2 & 1,2 \\
Preciso e não tenho & 3 & 1,8 \\
Não sei & & \\
\hline
\end{tabular}

Tabela 3. Dor de dente e absenteísmo declarados pelos funcionários de uma empresa alimentícia em Linhares, ES, 2007.

\begin{tabular}{|c|c|c|}
\hline Característica & $\mathbf{n}$ & $\%$ \\
\hline \multicolumn{3}{|l|}{ Dor espontânea ou provocada } \\
\hline Sim & 79 & 46,7 \\
\hline Não & 90 & 53,3 \\
\hline \multicolumn{3}{|l|}{ Visitas ao dentista em caso de dor } \\
\hline Sim & 45 & 57,0 \\
\hline Não & 34 & 43,0 \\
\hline \multicolumn{3}{|l|}{ Tipo de serviço utilizado } \\
\hline Empresa & 22 & 48,9 \\
\hline Unidade de saúde & 4 & 8,9 \\
\hline Particular & 18 & 40,0 \\
\hline Prático & 1 & 2,2 \\
\hline \multicolumn{3}{|l|}{ Número de visitas ao dentista } \\
\hline Uma vez & 18 & 40,0 \\
\hline Duas vezes & 9 & 20,0 \\
\hline Três ou mais vezes & 18 & 40,0 \\
\hline \multicolumn{3}{|c|}{ Motivo da não utilização serviço odontológico } \\
\hline Não sentiu necessidade & 31 & 91,3 \\
\hline Falta de dinheiro & 2 & 5,8 \\
\hline Medo & 1 & 2,9 \\
\hline \multicolumn{3}{|c|}{ Precisou faltar ao trabalho por dor de dente } \\
\hline Sim & 10 & 12,7 \\
\hline Não & 69 & 87,3 \\
\hline \multicolumn{3}{|l|}{ Tempo de falta ao trabalho } \\
\hline Meio-turno de trabalho & 6 & 60,0 \\
\hline Um turno de trabalho & 4 & 40,0 \\
\hline \multicolumn{3}{|c|}{ Compareceu ao trabalho com dor de dente } \\
\hline Sim & 38 & 48,1 \\
\hline Não & 41 & 51,9 \\
\hline
\end{tabular}


Entre os trabalhadores que declararam sentir dor de origem dentária, $48,1 \%$ compareceram ao trabalho com dor (Tabela 5).

\section{Discussão}

A dor de dente ainda se configura como um problema de saúde pública nas diversas regiões do Brasil, apesar da inserção da odontologia na Estratégia Saúde da Família e do aumento do investimento no programa Brasil Sorridente. As diretrizes de saúde bucal consideram como prioridade absoluta os casos de dor, infecção e sofrimento, valorizando o alto impacto produzido por estes eventos ${ }^{15}$. Ainda assim, os resultados encontrados no último inquérito nacional demonstraram que a dor ainda motivou a visita ao serviço odontológico de $17,3 \%$ da população de 35 a 44 anos da região sudeste. Estranhamente, quase a metade da população utilizou o serviço odontológico privado gerando uma inquietude em relação à resolutividade do serviço público ou a inexistência de horários alternativos que permitam o acesso da população trabalhadora. Pesquisas devem ser direcionadas para esclarecer essas questões ${ }^{16}$.

A prevalência de dor dentária entre os participantes deste estudo foi de 46,7\%, resultados similares aos encontrados em Toronto, Canadá

Tabela 4. Associação entre dor dentária e características sociodemográficas de trabalhadores dos funcionários de uma empresa alimentícia em Linhares, ES, 2007.

\begin{tabular}{|c|c|c|c|c|c|c|}
\hline \multirow[t]{2}{*}{ Característica } & \multicolumn{2}{|c|}{ Sentiu dor } & \multicolumn{2}{|c|}{ Não sentiu dor } & \multirow[t]{2}{*}{ p-valor } & \multirow[t]{2}{*}{ Odd Ratio } \\
\hline & $\mathbf{n}$ & $\%$ & $\mathbf{n}$ & $\%$ & & \\
\hline \multicolumn{7}{|l|}{ Sexo } \\
\hline Masculino & 48 & 42,9 & 64 & 57,1 & \multirow[t]{2}{*}{0,104} & 1,589 \\
\hline Feminino & 31 & 54,4 & 26 & 45,6 & & $0,836-3,021$ \\
\hline \multicolumn{7}{|l|}{ Faixa etária } \\
\hline Até 40 anos & 56 & 44,8 & 69 & 55,2 & \multirow[t]{2}{*}{0,248} & 1,349 \\
\hline 41 anos ou mais & 23 & 52,3 & 21 & 47,7 & & $0,827-2,688$ \\
\hline \multicolumn{7}{|l|}{ Renda familiar } \\
\hline Até $2 \mathrm{SM}$ & 39 & 50,0 & 39 & 50,0 & \multirow[t]{2}{*}{0,264} & 1,275 \\
\hline Mais de $2 \mathrm{SM}$ & 40 & 44,0 & 51 & 56,0 & & $0,695-2,339$ \\
\hline \multicolumn{7}{|l|}{ Escolaridade } \\
\hline Até ensino médio completo & 10 & 35,7 & 18 & 64,3 & \multirow[t]{2}{*}{0,150} & 1,724 \\
\hline Ensino médio completo e acima & 69 & 48,9 & 72 & 51,1 & & $0,744-4,000$ \\
\hline
\end{tabular}

Tabela 5. Dados sobre absenteísmo por causa de dor dentária em trabalhadores de uma empresa alimentícia em Linhares, ES, 2007.

\begin{tabular}{|c|c|c|c|c|c|c|}
\hline \multirow[t]{2}{*}{ Característica } & \multicolumn{2}{|c|}{$\begin{array}{c}\text { Perdeu horas } \\
\text { de trabalho } \\
\end{array}$} & \multicolumn{2}{|c|}{$\begin{array}{c}\text { Não perdeu horas } \\
\text { de trabalho }\end{array}$} & \multirow{2}{*}{ p-valor } & \multirow{2}{*}{ Odd Ratio } \\
\hline & $\mathbf{n}$ & $\%$ & $\mathbf{n}$ & $\%$ & & \\
\hline \multicolumn{7}{|l|}{ Sexo } \\
\hline Masculino & 7 & 14,6 & 41 & 85,4 & \multirow[t]{2}{*}{0,102} & 2,398 \\
\hline Feminino & 9 & 29,0 & 22 & 71,0 & & $0,785-7,299$ \\
\hline \multicolumn{7}{|l|}{ Faixa etária } \\
\hline Até 40 anos & 10 & 17,9 & 46 & 82,1 & \multirow[t]{2}{*}{0,296} & 1,623 \\
\hline 41 anos ou mais & 6 & 26,1 & 17 & 73,9 & & $0,511-5,154$ \\
\hline \multicolumn{7}{|l|}{ Renda familiar } \\
\hline Até $2 \mathrm{SM}$ & 10 & 25,6 & 29 & 74,4 & \multirow[t]{2}{*}{0,185} & 1,954 \\
\hline Mais de $2 \mathrm{SM}$ & 6 & 37,5 & 34 & 54,0 & & $0,633-6,030$ \\
\hline \multicolumn{7}{|l|}{ Escolaridade } \\
\hline Até ensino médio completo & 6 & 60,0 & 4 & 40,0 & \multirow[t]{2}{*}{0,004} & 8,850 \\
\hline Ensino médio completo e acima & 10 & 14,5 & 59 & 93,7 & & $2,114-37,046$ \\
\hline
\end{tabular}


$(39,7 \%)^{17}$, em Johore Bahru 43,6\% ${ }^{18}$ e na Austrália $51,9 \%{ }^{19}$.

Estudos realizados no Estado do Espírito Santo também apresentaram alta frequência de dor de dente nos últimos seis meses anteriores à data da pesquisa: em Venda Nova do Imigrante, com funcionários públicos municipais foi encontrada uma prevalência de $43 \%{ }^{4}$; em Marataízes, em pesquisa também com funcionários municipais, a prevalência encontrada foi de $57 \%{ }^{5}$. Os funcionários de um Hospital Universitário, localizado em Vitória, declararam uma prevalência de $65,7 \%{ }^{6}$. Todos estes resultados superam os encontrados nos dois últimos inquéritos nacionais. O estudo de 2003 revelou uma prevalência de dor nos últimos seis meses de $35,4 \%$ para a faixa etária de 15 a 19 anos e de $34,8 \%$ para adultos de 35 a 44 anos $^{3}$. Os resultados publicados para o Brasil em 2011 mostraram frequência de $24,7 \%$ e de 27,5\% para as faixas de 15 a 19 anos e de 35 a 44 anos respectivamente. Para a região sudeste os valores correspondem a 23,3\% e 30,8\% ${ }^{16}$. Em uma amostra de adolescentes residentes em Recife, $33,6 \%$ declararam ter sentido dor nos últimos seis meses ${ }^{11}$; no Rio de Janeiro, uma amostra de jovens puerperais registrou uma prevalência de dor dentária de $39,1 \%{ }^{20}$; em Florianópolis (SC), $33,7 \%$ dos escolares de 12 e 13 anos de idade declararam sentir dor dentária ${ }^{21}$, já os jovens de 18 anos do sexo masculino relataram uma frequência de $18,7 \%{ }^{22}$; em Porto Alegre, em uma amostra de adultos, constatou-se $20,7 \%{ }^{23}$; e em Natal, $29,6 \%{ }^{24}$

Chama a atenção a alta prevalência de dor orofacial $(66,1 \%)$ encontrada em trabalhadores do setor metalúrgico e mecânico em Xanxerê, $\mathrm{SC}^{25}$. As comparações entre esses estudos têm limitações devido a diferenças na faixa etária dificultadas pelos diversos pontos de corte na formação dos grupos - e a variações no período considerado para avaliar a frequência da dor, que variou de 15 dias a 12 meses, nos diversos estudos citados. Já os estudos realizados no Espírito Santo, utilizaram o mesmo roteiro e a mesma periodicidade de seis meses, favorecendo as comparações ${ }^{4-6}$. Desse modo, os resultados capixabas fazem soar um alerta, considerando as populações avaliadas, compostas de trabalhadores municipais e de um hospital universitário. O município de Linhares, local da realização desta pesquisa, configura-se como o de maior porte populacional do norte do estado. Possuía no momento da coleta de dados 23 Unidades de Saúde com serviço odontológico. Entretanto, nenhuma delas ofertava o terceiro turno de atendimento, horário alternativo que possibilitaria o acesso do trabalhador ao tratamento de rotina, sem prejuízo de suas atividades laborais. A indústria pesquisada, uma das maiores da região, possuía serviço odontológico para atendimento destinado aos seus trabalhadores. Este fato demonstra uma contradição dos resultados considerando a alta prevalência de dor de dente encontrada. Reconhece-se que a utilização do serviço odontológico pode ser influenciada por uma série de variáveis e que a disponibilidade de serviços nem sempre é capaz de gerar um impacto sobre essa utilização. Outros fatores podem estar envolvidos como a percepção da necessidade, além de fatores culturais e crenças em saúde. Pode-se considerar como limitação deste estudo a não investigação da estrutura da prática realizada pelo profissional da empresa, se preventiva, curativa, de urgência ou mutiladora. Não se conhece também a condição bucal desses trabalhadores no momento da admissão, o que reforça a necessidade do exame odontológico admissional, bem como do periódico. Necessário a realização de estudos direcionados a esta temática.

A amostra deste estudo apresenta particularidades: é essencialmente masculina, muito jovem, com boa escolaridade, esta não traduzida em renda. Metade dos participantes declarou não ter perdido nenhum dente, mesmo assim uma alta prevalência de dor esteve presente. É consenso que escolaridade é uma variável que tem mostrado associação com saúde bucal e utilização de serviços odontológicos. O impacto produzido por dor de dente envolve desde perda de horas de sono, diminuição da atenção, queda na produtividade, aumento do risco de acidentes laborais $^{26}$ até a ausência no local de trabalho ${ }^{4-6,8,21}$. Problemas relacionados à mastigação e dor de dente podem ser considerados fatores determinantes do bem estar oral da população de 35 a 44 $\operatorname{anos}^{27}$. A experiência da doença bucal é mais deletéria na percepção do adulto jovem do que nos idosos $^{28}$. Das condições bucais a dor de dente é a que afeta mais a qualidade de vida ${ }^{29}$.

Nenhuma das variáveis testadas neste estudo esteve relacionada à prevalência de dor de dente entre os participantes. Um estudo realizado no Espírito Santo mostrou associação com baixa escolaridade $^{4}$. Pesquisa desenvolvida no hospital Universitário em Vitória, ES, demonstrou uma associação com renda e condição socioeconômica: trabalhadores com menor renda e de condição socioeconômica menos favorecida tiveram maior prevalência de dor de dente ${ }^{6}$. Funcionários municipais de Marataízes, ES, de classe eco- 
nômica D e E também apresentaram maior prevalência de dor ${ }^{5}$. Resultados semelhantes foram relatados em outras pesquisas nacionais ${ }^{30-33}$.

Neste estudo, constatou-se que o problema da dor de dente foi suficientemente relevante para afastar do trabalho 10 (12,7\%) indivíduos dos que declararam dor nos últimos seis meses, resultado menor, quando comparado com outros estudos. Uma empresa que possui serviço odontológico deveria possibilitar acesso ao tratamento preventivo e restaurador, impedindo episódios de dor sem prejuízo das atividades laborativas e sem necessidade de deslocamento.

Em Venda Nova do Imigrante, ES, 17 (23,6\%) funcionários públicos municipais ${ }^{4}$ faltaram ao trabalho; em Marataízes, ES, 29 (17,8\%) servidores municipais relataram absenteísmo ${ }^{5}$; entre os funcionários de um Hospital Universitário em Vitória, ES, 75 (28,2\%) declararam falta do trabalho $^{6}$. Em pesquisa realizada em uma empresa agropecuária de Minas Gerais, 33\% dos trabalhadores declararam absenteísmo por causas odontológicas $^{34}$. Trabalhadores $(23,09 \%)$ de uma agroindústria em Curitiba também declararam absenteísmo por dor de dente ${ }^{35}$. Em Joaçaba, SC, foi verificada uma prevalência de absenteísmo por motivo odontológico de $16,2 \%{ }^{30}$. Quatro anos depois, trabalhadores (15\%) dessa mesma indústria declararam falta ao trabalho por dor de dente, um declínio praticamente inexistente ${ }^{8}$. Visitas ao serviço odontológico por motivo de urgência foram responsáveis por $25 \%$ de falta ao trabalho na Inglaterra ${ }^{36}$. Funcionários públicos do Rio de Janeiro (2,9\%) relataram a dor dentária como fator de impedimento para a realização de tarefas habituais, diferença esta explicada pelo fato de o período avaliado ter sido nas últimas duas semanas anteriores à coleta dos dados ${ }^{37}$. Já os realizados no Espírito Santo e os de Joaçaba, SC, avaliaram a frequência de dor e absenteísmo nos últimos seis meses ${ }^{8,31}$. Em Xanxerê, SC, foi verificada uma baixa prevalência de absenteísmo de $9,3 \%$, e surpreendente prevalência de dor orofacial $(66,1 \%)^{25}$

Funcionários com menor grau de escolaridade apresentaram maior relato de absenteísmo por dor dentária, corroborando outros estu$\operatorname{dos}^{4,5,7,8,31,36}$.

Este estudo encontrou uma taxa de $48 \%$ de presenteísmo, ou seja, quase a metade dos indivíduos declararam ter trabalhado com dor de dente, o que é um paradoxo, considerando a existência do serviço odontológico dentro da empresa, pois dor durante as atividades laborais gera perda da concentração no trabalho ${ }^{19}$, diminuição na produtividade $^{18}$ e até acidentes ${ }^{26}$. Este estudo vai ao encontro dos achados de Tauchen ${ }^{35}$ (59,48\%) e de Miotto et al. ${ }^{4}$ (50\%), em que uma grande massa dos trabalhadores relatou já ter trabalhado com dor dentária. Mais pesquisas envolvendo a associação entre comparecimento ao trabalho, dor de dente e acidentes de trabalho são necessárias para planejar serviços odontológicos que garantam o acesso resolutivo aos trabalhadores. Uma pesquisa adicional poderia revelar dados importantes a respeito do processo de trabalho do cirurgião-dentista na empresa estudada.

Este estudo encontrou uma taxa de utilização de serviços odontológicos de 57\% naqueles indivíduos que se queixavam de dor de dente nos últimos seis meses; $48,9 \%$ utilizaram o serviço ofertado pela empresa e $40 \%$ o serviço privado, utilização considerada alta, quando comparada com outros estudos. Locker e Grushka ${ }^{17}$ relataram que, para alívio da dor dentária, 44\% da amostra pesquisada procuraram serviço odontológico. A pesquisa de Jaafar et al. ${ }^{18}$ demonstrou que $38,1 \%$ dos sujeitos que declararam dor de dente não buscaram atendimento odontológico, esperando que a dor desaparecesse, o que sugere que dor dentária nem sempre é capaz de gerar demanda ${ }^{38}$.

Mais atenção deve ser dada ao desenvolvimento da política de saúde para reduzir as barreiras ao cuidado odontológico, o que inclui a expansão de horas de atendimento fora do horário de trabalho, reestruturação dos benefícios dos planos de saúde e instalação de clínicas no local de trabalho ${ }^{39}$.

Como muitas outras condições de saúde, as doenças bucais têm um forte componente social e comportamental. A importância de fatores sociais em Odontologia é evidente, especialmente em estudos que envolvem a utilização de serviços. Esta permanece altamente relacionada com sexo, idade, condição socioeconômica e estrutura do sistema ${ }^{38,40}$.

Dos participantes que receberam atendimento odontológico por dor dentária, 60\% visitaram o serviço duas ou mais vezes. Indivíduos com padrão irregular de visita ao serviço odontológico ou aqueles que só procuram atendimento por motivo de urgência provavelmente irão ficar mais tempo afastados das suas atividades laborais ou irão trabalhar com dor, maximizando o risco de acidentes de trabalho por falta de concentração. As empresas deveriam incentivar seus trabalhadores a realizar exame periódico odontológico, evitando episódios de urgência e falta ao trabalho, gerando economia, preservan- 
do a saúde bucal e melhorando a qualidade de vida de seus trabalhadores. Empresas com serviço odontológico resolutivos ou que garantam acesso por meio de convênios podem minimizar os efeitos nocivos da falta do terceiro turno de atendimento no serviço público, o que impede a utilização, em muitas regiões do Brasil, de trabalhadores que dependem do SUS. Mais estudos devem ser realizados para entendimento do efeito positivo provocado pela oferta de serviços odontológicos pelas empresas.

\section{Conclusão}

A prevalência de dor foi muito expressiva e não esteve associada às variáveis controladas por este estudo. A dor de dente observada foi capaz de produzir absenteísmo, e este esteve associado à escolaridade dos participantes. Os resultados encontrados demonstraram que mais importante que a presença do dentista nas empresas é a ne- cessidade de mudança da prática, priorizando ações de promoção de saúde. A realização de exames periódicos odontológicos deve ser incentivada com o objetivo de diagnóstico e intervenção precoce, minimizando os episódios de dor de dente que tanto impactam a qualidade de vida dos trabalhadores.

\section{Colaboradores}

MHMB Miotto participou da concepção da pesquisa, na metodologia, análise dos dados e na redação final do artigo. ZV Lopes participou da coleta de dados e da redação final do artigo. LA Barcellos participou da concepção da pesquisa, na metodologia, análise dos dados e na redação final do artigo

\section{Referências}

1. Sternbach R. Survey of pain in the United States: The Nuprin pain report. Clin J Pain 1986; 2(1):4953.

2. Sheiham A. Oral health, general health and quality of life. Bull World Health Organ [serial on the Internet].2005 Sept [cited 2007 Mar 7];83(9) Available from: http://www.scielosp.org/scielo.php?pid= 0042-968620050009\&script $=$ sci_issuetoc

3. Brasil. Ministério da Saúde (MS). Condições de saúde bucal da população brasileira 2002-2003: resultados principais. Brasília: MS; 2005.

4. Miotto MHMB, Silotti JCB, Barcellos LA. Dor dentária como motivo de absenteísmo em uma população de trabalhadores. Cien Saude Colet 2012; 17(5): 1355-1361.

5. Lima WJG. Dor dentária como causa de absenteísmo nos funcionários públicos da prefeitura municipal de Marataízes [monografia]. Vitória: Associação Brasileira de Odontologia; 2009.

6. Borgo PV. Prevalência de dor dentária e absenteísmo em trabalhadores do Hospital Universitário Cassiano Antônio de Moraes [monografia]. Vitória: Associação Brasileira de Odontologia; 2011.

7. Gift H, Reisine ST, Larach DC. The social impact of dental problems and visits. Am J Public Health 1992; 82(12):1663-1668.
8. Nardi A, Michel-Crosato M, Biazevic MGH, Cosato E, Pizzatto E, Queluz DP. Relationship betweewn orofacial pain and absenteeism among workers in Southern Brazil. Braz J Oral Sci 2009; 8(1):50-54.

9. Lima JRS. Absenteísmo por causa odontológica: análise comparativa entre funcionários da Prefeitura do Município de São José dos Campos e segurados do Instituto Nacional de Previdência Social: Inamps, 2001. Medcenter.com ${ }^{\circledR}$ Odontologia [periódico na internet]. 2001 [acessado 2007 maio 12]; Disponível em: http://www.odontologia.com.br/ artigos.asp? $\mathrm{id}=22$ \&idesp $=12$ \&ler $=\mathrm{s}$

10. Shepherd M, Nadanovsky PE, Sheiham A. The prevalence and impact of dental pain in 8-year-old schoolchildren in harrow. Br Dent J 1999; 187(1):38-41.

11. Goes PSA, Watt RG, Hardy R, Sheiham A. The prevalence and severity of dental pain in 14-15 years old Brazilian schoolchildren. Community Dent Health 2007; 24(4):217-224

12. Goes PSA, Watt RG, Hardy R, Sheiham A. Impacts of dental pain on daily activities of adolescents aged 14-15 years and their families. Acta Odontol Scand 2008; 66(1):7-12 
13. Miotto MHMB, Loureiro CA. Efeito das características sociodemográficas sobre a freqüência dos impactos dos problemas de saúde bucal na qualidade de vida. UFES Rev Odontol 2003; 5(2):6-13.

14. Brasil. Resolução no $196 / 96$, de 10 de outubro de 1996. Dispõe sobre as diretrizes e normas regulamentadoras de pesquisas envolvendo seres humanos. Diário Oficial da União 1996; 11 out.

15. Brasil. Ministério da Saúde (MS). Diretrizes de Saúde Bucal. Brasília: MS; 2004.

16. Brasil. Ministério da Saúde (MS). Pesquisa Nacional de Saúde Bucal: resultados principais. Brasília: MS; 2011.

17. Locker D, Grushka M. The Impact dental and facial pain. J Dent Res 1987; 66(9):1414-1417.

18. Jaafar N, Rasak IA, Zain RB. The impact of oral and pain in an industrial population. Ann Acad Med 1989; 18(5):553-555.

19. Sanders AEE, Spencer AJ. Job characteristics and the subjective oral health of Australian workers. Aust N Z J Public Health 2004; 28(3):259-266.

20. Vieira BHOM. Prevalência e impacto da dor de dente em uma população de mulheres grávidas no Rio de Janeiro, Brasil [tese]. Rio de Janeiro: Universidade do Estado do Rio de Janeiro; 2003.

21. Nomura LH, Bastos JLD, Peres MA. Prevalência de dor de dente e associação com cárie e condições socioeconômicas em escolares, sul do Brasil 2002. Braz Oral Res 2004; 18(2):134-140.

22. Bastos JLD, Nomura LHE, Peres MA. Dor de dente e sua relação com condições socioeconômicas e cárie dentária em adultos jovens do sexo masculino no Sul do Brasil. Cad Saude Publica 2005; 21(5):1416-1423.

23. Gomes AS. Avaliação dos impactos odontológicos no desempenho diário nos trabalhadores do departamento municipal de limpeza urbana de Porto Alegre [dissertação]. Porto Alegre: Universidade Federal do Rio Grande do Sul; 2005.

24. Ferreira AAA, Piuvezam G, Werner CWA, Alves MSCF. A dor e a perda dentária: representações sociais do cuidado à saúde bucal. Cien Saude Colet 2006; 11(1):211-218.

25. Lacerda JT, Traebert J, Zambenedetti ML. Dor orofacial e absenteísmo em trabalhadores da indústria metalúrgica e mecânica. Saude Soc 2008; 17(4):182191.

26. Gemeli TR. Aplicação e análise de metodologia investigatória de causalidade entre morbidez odontológica e acidentes de trabalho. In: Simpósio Nacional de Vigilância em Saúde do Trabalhador; 2005 jul; Florianópolis. [Documento da Internet]. 2005[acessado 2007 set 20]. Disponível em: http//: www.saude.sc.gov.br

27. Montero J, Bravo M, Vicente MP, Galindo MP, Lopez-Valverde A, Casals E, Cortes-Martinicorena FJ, Llodra JC. Oral pain and eating problems in Spanish adults and elderly in the Spanish National Survey perfomed in 2005. J Oralfac Pain 2011; 25(2):141-152.

28. Slade GD, Sanders AE. The paradox of better subjective oral health in older age. J Dent Res 2011; 90(11):1279-1285.
29. Pau A, Allen CD. Self-reported oral health status of adults resident in Medway, Kent in 2009. Prim Dent Care 2011;18(4):179-179.

30. Comunello MH. Dor dental e impacto nas atividades diárias: um estudo de prevalência em estudantes de 12 anos, Joaçaba e Herval d'Oeste, Brasil [dissertação]. Joaçaba: Universidade do Oeste de Santa Catarina; 2003.

31. Nardi A. Dor orofacial, absenteísmo e qualidade de vida em trabalhadores do sul do Brasil [dissertação]. Joaçaba: Universidade do Oeste de Santa Catarina; 2005.

32. Peres MA, Peres KG, Frias AC, Antunes JLF. Contextual and individual assessmente of dental pain perod prevalence in adolescents: a multilevel approach. BMC Oral Health 2010; 10(20):1-9.

33. Oliveira BA, Biazevic MGH, Michel-Crosato E. Prevalência de dor de dente, cárie dental e condições socioeconômicas: um estudo em adultos jovens brasileiros. Odonto 2011; 19(38):7-14.

34. Pereira MC, Oliveira MA, Araújo VE, Carvalho CM. Absenteísmo por causas odontológicas em uma empresa agropecuária da Região Sudeste do Estado de Minas Gerais. Rev Bras Pesq Saúde 2010; 12(1):1418.

35. Tauchen ALO. A contribuição do trabalho no programa de saúde ocupacional: verificando as condições de saúde bucal de trabalhadores de uma agroindústria do sul do Brasil [dissertação]. São Paulo: Universidade de São Paulo; 2006.

36. Reisine ST. Dental disease and work loss. J Dent Res 1984; 63(9):1158-1161.

37. Alexandre GC, Nadanovsky P, Lopes CS, Faerstein E. Prevalência e fatores associados à ocorrência da dor de dente que impediu a realização de tarefas habituais em uma população de funcionários públicos no Rio de Janeiro, Brasil. Cad Saude Publica 2006; 22(5):1073-1078.

38. Reisine ST. The impact of dental conditions on social functioning and the quality of life. Ann Rev Public Health 1988; 9:1-19.

39. Reisine ST, Miller J. A longitudinal study of work loss related to dental diseases. Soc Sci Med 1985; 21(12):1309-1314.

40. Barcellos LA, Loureiro CA. O público do serviço odontológico. UFES Rev Odontol 2004; 6(2):41-50.

Artigo apresentado em 02/08/2012

Aprovado em 10/10/2012

Versão final apresentada em 26/10/2012 